\title{
Diagnostic CT radiation and cancer induction
}

\author{
Paula J. Richards • Jennifer George
}

Published online: 2 December 2009

(C) ISS 2009

Background radiation makes up most of an individual's exposure; however, medical sources are the largest manmade component and have been rising rapidly [1-3]. Whilst in the UK general radiology doses have decreased dramatically [4], it is only interventional and CT doses that have increased. CT use in particular has increased, and in 7 years one US Level 1 Trauma Centre increased the use of chest CT from 2.7 to $28.7 \%$ of blunt trauma cases [5].

The deterministic effects of radiation occur after a predictable threshold dose, e.g. opacification in the lens of the eye is followed by cataracts, or skin irradiation progressively produces transient erythema, temporary epilation, skin erythema, and telangiectasia to desquamation at increasing threshold doses. Random stochastic effects, which are not dependent on the threshold, include genetic mutations and carcinogenesis. The effect of radiation from CT scans depends on the field of view scanned and the sensitivity of the organs within the area. Highly radiosensitive organs include bone marrow, lung, stomach mucosa, thyroid and breast glandular tissue. Thyroid irradiation may cause hypothyroidism and thyroid cancers, particularly at a

P. J. Richards $(\bowtie)$

X-ray Department,

University Hospital of North Staffordshire NHS Trust, Princes Road,

Hartshill, Stoke-on-Trent ST4 7LN Staffordshire, UK

e-mail: paula.richards@uhns.nhs.uk

P. J. Richards

Keele University,

Newcastle-under-Lyme, UK

J. George

Clinical Scientist Radiology Physics, Medical Physics Directorate, UHNS,

Stoke-on-Trent, UK younger age [6]. Atomic bomb survivors, exposed to up to 4 Gy, have shown a linear and statistically highly significant radiation dose response, causing breast cancers [7].

The Computed Tomography Dose Index (CTDI) works out concentration units, expressed in $\mathrm{mGy}$, for a procedure, depending on the total energy deposited in the patient divided by the mass of that section. Using Monte Carlo dosimetry [8], the CTDI value can be used to derive mean organ doses in a CT section in millisieverts ( $\mathrm{mSv})$. The effective dose (E) is the sum of the dose to individual organs, each organ dose being weighted according to the radiosensitivity, and can be converted numerically to estimate excess radiation risk. It has been recommended [9] that effective dose values be treated with caution as additional uncertainty is introduced by applying organ weighting factors, but nevertheless the effective dose quantity is a useful measure when received as an indication of relative total stochastic risk, that is, the random risk. The International Commission on Radiation Protection (ICRP) has set the nominal risk coefficient for cancer induction at $5.5 \%$ per sievert for adults [9]. Some feel that this relationship between dose and cancer risk is controversial [4].

There is a large variation in the calculated effective dose between X-ray units and CT scanners [10-12]. For example, in a recent UK national survey, the chest, abdomen and pelvis examination mean effective dose was 12 millisieverts ( $\mathrm{mSv}$ ) with a coefficient of variation of $34 \%$ for multislice scanners [10]. Variations in effective dose have also been reported for the same model of scanner because of protocol variations [13]. Modern multislice scanners are so fast that there is an inevitable tendency to scan a larger field of view (the length of the patient) than is absolutely necessary [3] and increased scanning of the whole cervical spine rather than level-specific examinations [14]. Estimation of life time cancer risks also varies widely 
over different countries [15]. Both national and international radiological protection organisations believe there to be a linear, cumulative and proportionate risk of causing a cancer over one's life time [16-18].

Hence, each medical exposure must be justified [19] and it is important that we optimise multislice techniques to answer the clinical question, limiting the CT to exactly what is required, minimising the radiation used to produce diagnostic images, with the patient dose as low as is reasonably practicable (the ALARP principle). Every bit of diagnostic information must be obtained from a single pass through the CT scanner, including dorsal and lumbar spine reconstructions from trauma body CTs [20-22] and coronal and sagittal pelvis reconstructions from abdomen and pelvis CT scans $[23,24]$. This obviates the need for dorsal and lumbar spine radiographs $[25,26]$. The streak artefact from dental amalgam, which may cause cervical fractures to be missed are readily seen on lateral cervical radiographs and should probably still be included [26]. Collaborations between radiologists [27] and medical physicists [20] help reduce the exposure without compromising image quality.

Optimised protocols on multislice scanners may be achieved by reducing the effective $m A$ s to the lowest value possible whilst maintaining adequate noise levels and by using the widest possible beam collimation consistent, for better geometrical efficiency, with the reconstruction required, and some dose reduction is achieved in angiography by reducing $\mathrm{kV}$ values [1]. In general, the lowest mAs values are achieved in areas of high inherent contrast such as imaging of bone or renal calculi [28] and similarly paranasal sinuses and the cervical spine, with no loss of diagnostic quality and so reducing the cancer risk [29].

The radiation dose from cervical spine CT gives a high dose to the thyroid, which is particularly radiosensitive [14, 30, 31]; thus risk stratification can reduce CT scanning by using in the UK, say, radiographs of conscious patients and CT in unconscious major trauma cases [32, 33]. In 2002, Rybicki et al. reported a 14-fold increase in radiation to the thyroid when changing from cervical radiography to CT of the whole cervical spine [31]. At our institution this is equivalent to $0.1 \mathrm{mSv}$ or one extra cancer in 200,000 for a three-film x-ray series of the cervical spine to $3.5 \mathrm{mSv}$ or 1:5,200 for CT of the cervical spine. Images of better quality can be obtained by increasing the pitch with a small slice thickness, rather than increasing the slice thickness with a lower pitch in cervical spine CT [34]. Optimal MCT cervical image quality is at $1.25-\mathrm{mm}$ slice thickness with a table speed of $7.5 / 360^{\circ}$ using a pitch of 1.5 and this is also faster than when a pitch of 0.75 is used [34]. Reducing the energy of the X-ray beam in cervical CT scans has a great effect on the radiation dose, that is, around 65\%: 6MCT 130 vs $110 \mathrm{kV}$ gives 23.17 vs $15.27 \mathrm{mGy}$ and $16 \mathrm{MCT} 120$ vs $100 \mathrm{kV}$ gives 19.48 vs
$12.48 \mathrm{mGy}$, without a subjective change in image quality [35]. (Gray [Gy] is an absolute measurement, in this case numerically equivalent to sieverts, but does not take into account organ radiosensitivity).

In our institution, the $\mathrm{CT}$ dose of the craniocervical junction was computed to be low $(0.3 \mathrm{mSv})$ and 10 -fold more if the whole cervical spine was scanned $(3.5 \mathrm{mSv})$ [14]. For one 16 MCT using the Monte Carlo software [8], whole dorsal spine $\mathrm{CT}$ has a lower risk of causing a cancer (10 $\mathrm{mSv}$ or 1:1,800 life time cancer risk) compared with $\mathrm{CT}$ of the chest, abdomen and pelvis $(\mathrm{ED}=12 \mathrm{mSv}$, risk $6 \times 10^{-4}$ or 1 in 1,500 with the current protocol and correction for dose modulation and overscan). Whole lumbar CT $(5.6 \mathrm{mSv}$ or $1: 3,200)$ is lower than that of the dorsal spine, partly because of the shorter length of the scan [36]. Scanning three lumbar vertebrae carries a similar risk to scanning three dorsal ones, but subtle variations occur because of different quantities of bone and from all the other structures in the slice, for instance, the iliac wings at L5, which will also absorb radiation [36]. Lumbar quantatitive $\mathrm{CT}$, on the other hand, involves a very low dose because after a scanogram, only three slices are obtained at L1, L2 and L3 $(0.1 \mathrm{mSv}$ or 1:200,000) [36]. Huda and Morin reported typical patient doses in bone mineral densitometry [37] of $2.5 \mu \mathrm{Sv}$ for DEXA and $300 \mu \mathrm{Sv}$ for quantatitive CT or $0.0025 \mathrm{mSv}$ and $0.3 \mathrm{mSv}$ respectively. For comparison, a single chest X-ray is typically $0.02 \mathrm{mSv}$ or 1:1,000,000 life time cancer risk.

It is more important to reconstruct CT of the chest, abdomen and pelvis for whole dorsal spine images, saving re-scans and its attendant radiation dose [20], than brain $\mathrm{CT}$, which is a relatively low dose study $(2 \mathrm{mSv})$, because the only particularly radiosensitive organ in the scan area is the thyroid and that is outside the main X-ray beam [30]. Cardiac coronary CT has a very high dose, but the use of dose modulation nearly halved the cancer risk from about $1: 114$ to $1: 219$ [38], and a further $54 \%$ may be saved by optimizing ECG gating [37]. Nevertheless, if a patient over a lifetime had a dorsal spine, a major trauma protocol, i.e. chest, abdomen, pelvis, whole cervical spine and brain, and subsequently a cardiac CT, then $1: 160$ or one additional cancer may be induced in the region of every 160 patients scanned that way $(\mathrm{D}$ spine $=1$ in $1,800, \mathrm{CAP}=1$ in 1,500 , whole $\mathrm{C}$ spine $=1$ in 5,200, CT brain $=1$ in 9,000, cardiac 1 in 219; add the risks and get approximately 1 in 160 or 0.00625). If that person had a second accident and areas are re-imaged and another cardiac CT then the risk is $1 / 160+1 /$ $160=0.0125$ or $1: 80$. It should be noted that this should be seen as very approximate as the different risks have come from different sources and using different risk coefficients.

Children up to the age of 15 years are the most vulnerable to radiation. Risk reduces rapidly up to 30 years, then plateaus, falling again after 60 years of age [39]. This 
means that clinicians need to consider the risk/benefit analysis of $\mathrm{CT}$ in relation to each patient and their age and the IR(ME)R regulations in the UK require special attention to be paid to optimisation of the medical exposure of children [19]. Clinicians generally have a poor understanding of radiation doses [40], let alone the effects of different body habitus, with the vulnerability of the small body of a child and the dramatic effect body size has on the radiation required to obtain diagnostic images [41]. Thirty centimetres of body fat can increase the CT dose 80 -fold, but it is reduced by orientating the patients thickest fat layer opposite the tube [42]. It is inherently obvious that the use of a well set-up dose modulation system, restricting the radiation appropriate to the patient's body habitus, be they size zero or bariatric, will reduce the cancer risk, by optimising the exposure [43]. It is important to reinforce to the clinicians that each examination must be justified clinically [19]; stressing that CT is a whole body screening tool is foolhardy [44]. Until an American ER physician is sued for causing a cancer, not missing a fracture, or liberal widespread imaging restricted in some manner, US practices are unlikely to change, but in health care systems where the radiologists are the gate keepers there is more hope of minimising cancer risk.

In conclusion, the risks of cancer induction from $\mathrm{CT}$ imaging of different parts of the body can be expressed as a simple risk ratio, which are additive and easily understood. These risk ratios, seen in the context of the large uncertainties involved, can be used to compare relative risk for different examinations and protocols, optimise our practice, allowing better communication among clinicians, radiologists and patients. For the future should we hope that MRI or a new non-radiation-based technology will replace CT or should we cumulatively $\log$ each individual's millisieverts, to encourage improved CT practices? There may be a place for internationally agreed $\mathrm{CT}$ protocols for each make and model, aiding dose optimisation.

\section{References}

1. Kalra MK, Maher MM, Toth TL, Hamberg LM, Blake MA, Shepard JA, et al. Strategies for CT radiation dose optimization. Radiology. 2004;230:610-28.

2. Crawley MT, Booth A, Wainwright A. A practical approach to the first iteration in the optimization of radiation dose and image quality in CT: estimates of the collective dose savings achieved. Br J Radiol. 2001;74:607-14.

3. Van der Molen AJ, Geleijns J. Quantification of overscanning and relative contribution to scan length and effective dose in 16-slice multislice CT. AJR Am J Roentgenol. 2004;182(4):27.

4. Hart D, Hillier MC, Wall BF. Doses to patients from radiographic and fluoroscopic $\mathrm{x}$-ray imaging procedures in the UK-2005 review, HPA-RPD-029.
5. Plurad D, Green D, Demetriades D, Rhee P. The increasing use of chest CT for trauma: is it being overused? J Trauma. 2007;62:631-5.

6. Hancock SL, McDougall R, Constine LS. Thyroid abnormalities after therapeutic external radiation. Int J Radiat Oncol Biol Phys. 1995;31(5):1165-70.

7. Land CE, Tokunaga M, Koyama K, Soda M, Preston DL, Nishimori $\mathrm{I}$, et al. Incidence of female breast cancer among atomic bomb survivors, Hiroshima and Nagasaki, 1950-1990. Radiat Res. 2003;160:707-17.

8. Jones DG, Shrimpton PC. Normalised organ doses for x-ray computed tomography calculated using Monte Carlo techniques. Chilton; 1993, NRPB-SR250.

9. IRCP(200)IRCP publication 103. The 2007 Recommendations of the International Commission on Radiological Protection, vol 37, No204.

10. Shrimpton PC, Hillier MC, Lewis, MA, Dunn, M. Doses from computed tomography (CT) examinations in the UK-2003 review. NRPB-W67, Didcot; 2005.

11. Koller CJ, Eatough JP, Bettridge A. Variations in radiation dose between the same model of multislice CT scanner at different hospitals. Br J Radiol. 2003;76:798-802.

12. Hausleiter J, Meyer T, Hermann F, Hadamitzky M, Krebs M, Gerber TC, et al. Estimated radiation dose associated with cardiac CT angiography. JAMA. 2009;301(5):500-7.

13. Tsapaki V, Aldrich Je, Sharma R, Staniszewska AM, Krisanachinda A, Rehani $M$, et al. Dose reduction in CT while maintaining diagnostic confidence: diagnostic reference levels at routine head, chest, and abdominal CT-IAEA coordinated research project. Radiology. 2006;240(3):828-34.

14. Chan PN, Antonio GE, Griffith JF, Yu KW, Rainer TH, Ahuja AT. Computed tomography for cervical spine trauma. The impact of MDCT on fracture detection and dose deposition. Emerg Radiol. 2005;11:286-90.

15. Gonzalez A, Darby S. Risk of cancer from diagnostic X-rays: estimates for the UK and 14 other countries. Lancet. 2004;363: 345-51.

16. Brenner DJ, Doll R, Goodland DT, Hall EJ, Land CE, Little JB, et al. Cancer risk attributable to low doses of ionising radiation: assessing what we really know. Proc Natl Acad Sci USA. 2003;100(24):13761-6.

17. NRCP report 136. Evaluation of the linear nonthreshold doseresponse model for ionising radiation. Bethesda: NRCP; 2001.

18. UNSCEAR 2000. Sources and effects of ionising radiation: vol 2 Effects. New York: UN; 2000.

19. IR(MER). The Ionising Radiation (Medical Exposure) Regulations 2000.SI (2000) No. 1059. TSO, London; 2002.

20. Wintermark M, Mouhsine E, Theumann N, Mordasini P, Ivan MG, Leyrraz PF. Thorocolumbar spine fracture in patients who have sustained severe trauma: depiction with MCT. Radiology. 2003;227:681-9.

21. Brown CVR, Antevil JL, Sise MJ, Sack DI. Spiral computed tomography for the diagnosis of cervical, thoracic and lumbar spine fractures: its time has come. J Trauma. 2005;58:890-6.

22. Hauser CJ, Visvikis G, Hinrichs C, Ener CD, Cho K, Lavery RF, et al. Prospective validation of computed tomographic screening of the thoracolumbar spine in trauma. J Trauma Injury. 2003;55:228-35.

23. Roos JE, Hifiker P, Platz A, Desbiolles L, Boehm T, Marineck B, Weishaupt D. MDCT in emergency radiology: is a standardised chest or abdominal protocol sufficient for evaluation of thoracic and lumbar spine trauma? AJR Am J Roentgenol. 2004;183:95968.

24. Berry GE, Adams S, Harris MB, Boles CA, McKerian MG, Collinson $\mathrm{F}$, et al. Are plain radiographs of the spine necessary during evaluation after blunt trauma? J Trauma. 2005;59:1410-3. 
25. Lucy BC, Stuhlfaut JW, Hochberg AR, Varghese JC, Soto JA. Evaluation of blunt abdominal trauma using PACS-Based 2D and 3D MDCT reformations of the lumbar spine and pelvis. AJR Am J Roentgenol. 2005;185:1435-40.

26. Nagel BG, HD SG, Veit R, Lechel U, Griebel J, Galanski M. Radiation exposure in multi-slice versus single-slice CT: results of nationwide survey. Eur Radiol. 2003;13:1979-91.

27. Daffner RH, Sciulli RI, Rodriquez A, Protetch J. Imaging for evaluation of suspected cervical spine trauma: a 2-year analysis. Injury. 2006;37:652-8.

28. Heggie JCP. Patient doses in multi-slice CT and the importance pf optimisation. Australas Phys Eng Sci Med. 2005;28(2):86-96.

29. Brem MH, Zamani AA, Riva R, Zou KH, Rumboldt Z, Hennig $\mathrm{FF}$, et al. Multidetector CT of the paranasal sinus: potential for radiation dose reduction. Radiology. 2007;243(3):847-52.

30. Richards PJ, Summerfield R, George J, Hamid A, Oakley P. Major trauma and cervical clearance radiation doses and cancer induction. Injury Int J Care Injured. 2008;39:347-56.

31. Rybicki F, Nawfel RD, Judy PF, Ledbetter S, Dyson RL, Halt PS, et al. Skin and thyroid dosimetry in cervical spine screening: two methods for evaluation and a comparison between a helical CT and radiographic trauma series. AJR Am J Roentgenol. 2002;179:933-7.

32. Royal College of Radiologists. Making the best use of a department of clinical radiology guidelines for doctors, 5th Ed; 2003.

33. NICE-National Institute for Clinical Excellence. Head injury: Triage, assessment, investigation and early management of head injury in infants, children and adults. Clinical Guideline 4 June 2003.

34. Obernauer S, Alamo L, Herold T, Funke M, Kopka L, Grabbe E. Imaging skeletal anatomy of injured cervical spine specimens: comparison of single-slice vs multi-slice helical CT. Eur Radiol. 2002;12:2107-11.

35. Mulkens TH, Marchal P, Daineffe S, Salgado R, Bellinck P, te Rijdt $\mathrm{B}$, et al. Comparison of low-dose multidetector $\mathrm{CT}$ in cervical spine trauma. AJNR Am J Neuroradiol. 2007;28:1444-51.

36. Richards PJ, George J, Metelko M. Spine CT doses and cancer induction. Spine 2009, In press.

37. Huda W, Morin RL. Patient doses in bone mineral densitometry. Br J Radiol. 1996;69:422-5.

38. Einstein AJ, Henzlova MJ, Rajagopalan S. Estimating risk of cancer associated with radiation exposure from 64-slice computed tomography coronary angiography. JAMA. 2007;298(3):317-23.

39. Weustink AC, Mollet NR, Neefjes LA, van Straten M, Neoh E, Kyropoulos S, et al. Preserved diagnostic performance of dualsource CT coronary angiography with reduced radiation exposure and cancer risk. Radiology. 2009;252(1):53-60.

40. Wall BF. Radiation protection dosimetry for diagnostic radiology patients. Radiat Prot Dosim. 2004;109(4):409-19.

41. Fazel R, Krumholz HM, Wang Y, Ross JS, Chen J, Ting HH, et al. Exposure to low-dose ionizing radiation from medical imaging procedures. N Engl J Med. 2009;361:849-57.

42. Yanch JC, Behrman RH, Hendricks MJ, McCall JH. Increased radiation dose to overweight and obese patients from radiographic examinations. Radiology. 2009;252(1):128-39.

43. Deak PD, Langner O, Lell M, Kalender WA. Effects of adaptive section collimation on patient radiation dose in multisection spiral CT. Radiology. 2009;252(1):140-7.

44. Marshall. A report by the clinical advisory committee on diagnostic imaging on the safety and efficiency of CT screening. 2007;1-28. 